\title{
JUNCUS SQUARROSUS (JUNCACEAE) IN ROMANIA: ITS FIRST CERTAIN OCCURRENCE AND A NEW PLANT ASSOCIATION FOR THE ROMANIAN VEGETATION
}

\author{
József PÁl Frink¹, Gheorghe Coldea, Andrea Sass-Gyarmati, \\ TAMÁs Pócs \& GuSZTÁV JAKAB
}

\begin{abstract}
This study confirms the first certain occurrence of Juncus squarrosus L., an Atlantic species, in the Romanian native flora. Characteristic plant communities with J. squarrosus occur in the Apuseni Mts (Western Romanian Carpathians). At these sites $J$. squarrosus has the southeastern limit of its European distribution. The identified plant communities with $J$. squarrosus in Romania are devoid of some Atlantic species present in Western and Central European communities and therefore are assigned to the Nardo-Juncetum squarrosi (Nordh. 1920) Bük. 1942 association. This plant community is a new syntaxon for the Romanian vegetation.
\end{abstract}

Key words: Atlantic species, Apuseni Mountains, distribution, heath, Nardo-Juncetum squarrosi association, phytosociology

József Pál Frink, National Institute for Research and Development in Forestry 'Marin Drăcea', Horea St. No. 65, 400275-ClujNapoca, Romania; e-mail: jpfrink@gmail.com

Gheorghe Coldea, Institute of Biological Research, National Institute of Research and Development for Biological Sciences, Republicii St., No. 48, 400015-Cluj-Napoca, Romania; e-mail: office@icbcluj.ro

Andrea Sass-Gyarmati \& Tamás Pócs, Institute of Biology, Eszterházy Károly University, Pf. 43, 3301-Eger, Hungary; e-mails: sassgyarmati@gmail.com,colura@upcmail.hu

Gusztáv Jakab, Szent István University, Tessedik Campus, Szabadság St., No. 1-3, 5540-Szarvas, Hungary; e-mail: cembra@ freemail.hu

\section{INTRODUCTION}

Juncus squarrosus L. (syn. Juncus ellmanii C. E. Hubb., Sandwith \& Turrill, J. sprengelii Willd., $J$. strictus Lucé) is a perennial caespitose plant with rigid stems $15-50 \mathrm{~cm}$ long and many basal subcoriaceous leaves forming dense tufts (Fig. 1). This species is widespread in the British Isles and in oceanic parts of Europe; it is considered an Atlantic floristic element (Welch 1966a; Ellenberg 1979). Its distribution area also extends to the south of Spain and Central and Eastern Europe to the Dnieper River, being present in scattered localities (Meusel et al. 1965; Welch 1966a; Snogerup 1980; Andrienko 1983). It is also found in southern Greenland as well as in Iceland, Svalbard and Scandinavia, especially in the western oceanic areas (Meusel et al. 1965; Welch 1966a; Anonymous

\footnotetext{
1 Corresponding author
}

2006-2017). It occurs abundantly on siliceous bedrock in oligotrophic grasslands, heaths, moors and bogs, where it is the dominant and characteristic species in some communities of OxycoccoSphagnetea Br.-B1. \& Tx. 1943 (Ericion tetralicis Schwickerath 1933), Calluno-Ulicetea Br.-B1. \& R. Tx. ex Klika \& Hadač 1944 (Juncion squarrosi Oberd. 1956; Rodwell et al. 2002) and NardoCallunetea Prsq. 1949 (Nardo-Juncion squarrosi (Oberd. 1957) Passarge 1964; Oberdorfer 1978). According to Welch (1964, 1966a, b), J. squarrosus has strong reproductive capability due to its large production of viable seeds and good vegetative development. It is unpalatable in summer, but the inflorescences are grazed (Welch 1964, 1966b).

In Romania the presence of $J$. squarrosus has been considered uncertain until now. Although it has been mentioned since the $19^{\text {th }}$ century, its 

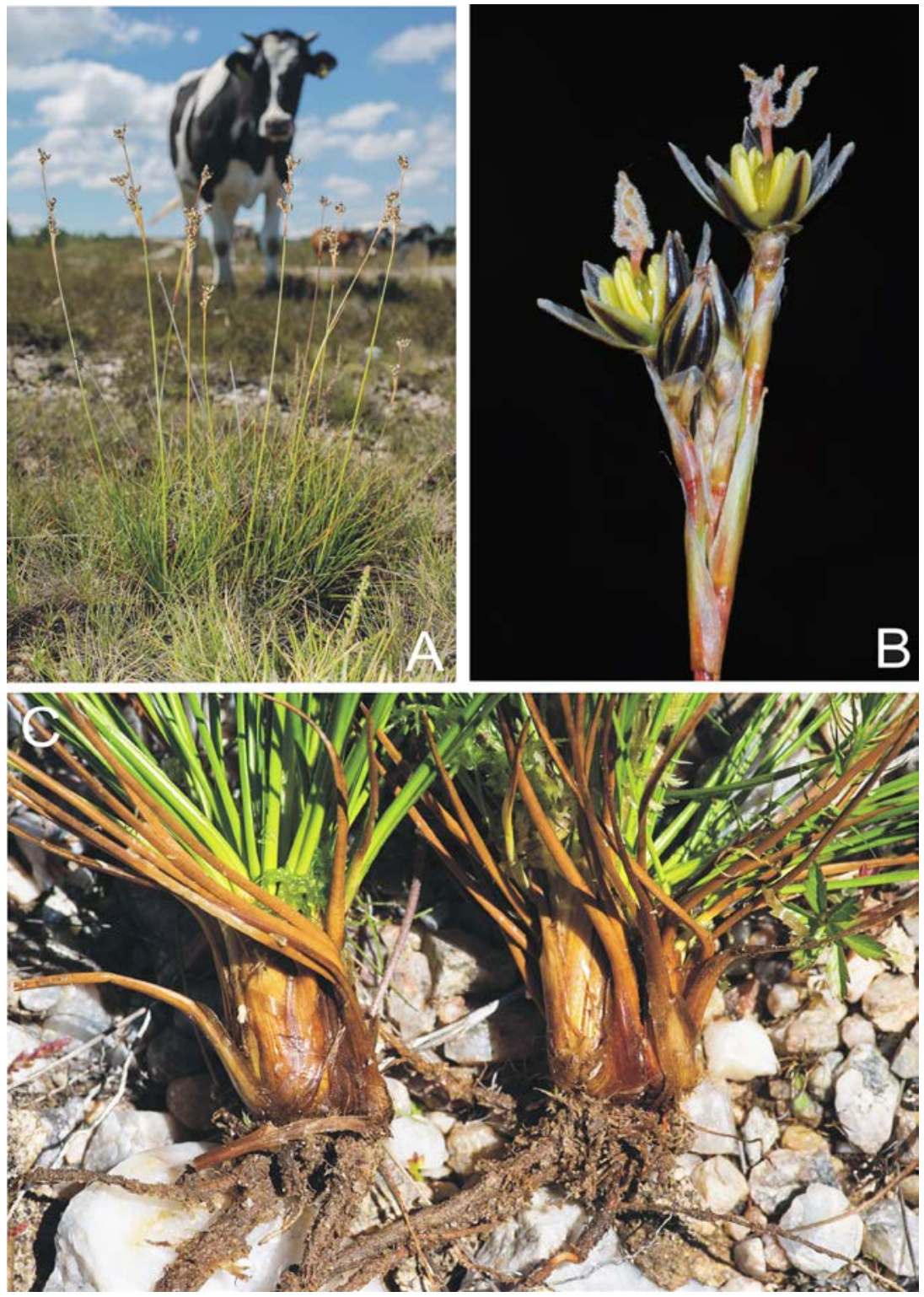

Fig. 1. Juncus squarrosus L. A - habit, B - inflorescence, C - tufts. Photo G. Jakab.

occurrence has always been contested because of the lack of herbarium material. The earliest mention of this species in Transylvania is found in Baumgarten`s study (1816, En. 3: 335) under the name Juncus squarrosus Wild. Later this species was mentioned by Steffek (1864), who found it 'common' in the surroundings of Oradea (Hungarian: Nagyvárad; German: Grosswardein).
Neilreich (1866) discussed the species mentioned by Steffek but cast doubt on the accuracy of the taxon identification. Since Baumgarten did not attribute the description of this species to Linné, Simonkai (1886) reconsidered Baumgarten`s taxon and related it with Juncus compressus Jacq. (Juncus squarrosus non L., Baumg. En. 3: 335. 1816). Moreover, in his study from 1890, Simonkai 
eliminated $J$. squarrosus from the list of plants to be found around Oradea.

At the beginning of the $20^{\text {th }}$ century, Prodan (1939), based only on bibliographic data, mentioned this species in the northwestern part of Romania (Oradea) as well as in Moldova and the Ciuc Basin (Eastern Carpathians). Because there was no herbarium material in the botanists' early $20^{\text {th }}$ century studies, the occurrence of $J$. squarrosus in the Transylvanian and Romanian flora was considered questionable (Jávorka 1925; Soó 1940; Borza 1947; Grinţescu 1966). That is why $J$ squarrosus is not mentioned in Flora Europaea (Snogerup 1980) as occurring in Romania. Nor is the species reported from Romania in floristic monographs recently published by Romanian botanists (Ciocârlan 2000, 2009; Oprea 2005; Chifu et al. 2006; Sârbu et al. 2013).

This paper reports the first certain occurrence of J. squarrosus in the Romanian native flora, and analyzes the floristic composition of plant communities in which the species occurs.

\section{STUDY AREA, MATERIAL AND METHODS}

During vegetation studies in 2013 in the Apuseni Mountains, J. squarrosus was identified close to Beliş and Dealu Negru villages (Frink et al. 2014). This area is part of a larger erosional mountain plateau ( $c a 1100 \mathrm{~m}$ a.s.1.), formed on crystallized deposits which date to the Paleozoic, covered by paleogene sediments (Pop 1962) and having a slight inclination $\left(5^{\circ}-15^{\circ}\right)$. The geographic, geologic and topoclimatic features of this tableland facilitate the development of bogs. Oligotrophic bogs occur to various extents in the whole area (Pop 1947, 1960).

Herbarium vouchers with $J$. squarrosus are deposited in the Herbarium of Babeş-Bolyai University, Cluj-Napoca (CL, inventory no. 664521, leg. Frink J.P., Jakab G., Sass-Gyarmati A.) and in the Herbarium of Eszterházy Károly University, Eger (EGR, inventory nos 8152, 8153, leg. Frink J.P., Jakab G., Sass-Gyarmati A. and no. 14110/C, leg. Höhn M., Pócs T., Tóth E.).

Between 2014 and 2016, additional field surveys were made in order to investigate the local ecological conditions of $J$. squarrosus and its phytosociological characteristics. Also, the neighboring areas of its occurrence (Mărişel area, Stâna de Vale area and Beiuş Depression in the western part of the Apuseni Mts) were thoroughly surveyed in order to discover new localities of it.

The field survey concerning the floristic structure of plant communities with $J$. squarrosus followed the Braun-Blanquet method $(1928,1964)$, with modifications proposed by Borza (1934). These modifications entail using the local frequency of each species in the sample plot (phytosociological relevé), instead of considering their sociability. This substitution readily reveals the local spread of each species within the sample plot. The species registered in the relevés were entered in a synthetic table and grouped, according to their ecological and phytosociological characteristics, in diagnostic species for alliances, orders and vegetation classes. This floristical-ecological criterion was used to establish more accurately the position of plant communities with $J$. squarrosus from Romania in the Central European syntaxonomical system (Passarge 1964; Oberdorfer 1978; Krahulec et al. 2007; Matuszkiewicz 2008). Nomenclature of vascular plants follows Flora Europaea (Tutin et al. 1964-1980), and that of bryophytes follows Hill et al. (2006).

\section{RESULTS AND DISCUSSION}

The Juncus squarrosus populations are found in the Molhaşul de la Rîşca (Mohuşul de la Dealul Negru - Lágyas), Dîmbul Negru - La Pod and Dîmbul Negru - Platou oligotrophic bogs (local toponymy according to Pop 1947, 1960). At these sites $J$. squarrosus has the southeastern limit of its European distribution. During the field research between 2014 and 2016 in the neighboring areas of its occurrence, no other new localities with $J$ squarrosus were identified.

The plant communities with $J$. squarrosus are patchily distributed on small surfaces $\left(20-30 \mathrm{~m}^{2}\right)$, mainly on the edge of flat bogs (blanket bogs). They come into contact with hygrophilous coenoses of the Epilobio-Juncetum effusi Oberd. 1957 association and with oligotrophic coenoses of the Vaccinio-Callunetum Bük. 1942 community. The soil on which the phytocoenoses with J. squarrosus occur is reddish brown peaty gley with excess moisture most of the year, and has an acid reaction (according to our field measurement, $\mathrm{pH}$ 4.9-5.5). This species is considered by Ellenberg (1979) to be an oceanic, heliophilous, hygrophilous and 
Table 1. Floristic composition of Nardo-Juncetum squarrosi (Nordh. 1920) Bük. 1942 association in Romania.

\begin{tabular}{|c|c|c|c|c|c|c|c|c|c|c|c|c|c|c|c|c|}
\hline Relevé no. & 1 & 2 & 3 & 4 & 5 & 6 & 7 & 8 & 9 & 10 & 11 & 12 & 13 & 14 & 15 & \multirow{7}{*}{$\mathrm{F} \%$} \\
\hline Elevation $(\times 10 \mathrm{~m}$ a.s.1. $)$ & 106 & 106 & 108 & 108 & 108 & 109 & 110 & 110 & 110 & 109 & 106 & 108 & 110 & 109 & 110 & \\
\hline Aspect & $\mathrm{N}$ & NW & W & W & $\mathrm{W}$ & NW & W & - & - & W & $\mathrm{S}$ & - & - & - & - & \\
\hline Slope $\left(^{\circ}\right)$ & 2 & 2 & 2 & 2 & 1 & 3 & 3 & - & - & 3 & 3 & - & - & - & - & \\
\hline Sample plot size $\left(\mathrm{m}^{2}\right)$ & 16 & 16 & 16 & 16 & 16 & 16 & 16 & 16 & 16 & 16 & 16 & 16 & 16 & 16 & 16 & \\
\hline Grass cover $(\%)$ & 55 & 50 & 90 & 100 & 95 & 100 & 100 & 95 & 95 & 80 & 90 & 80 & 70 & 85 & 90 & \\
\hline Moss cover (\%) & 10 & 7 & 40 & 50 & 1 & 60 & 45 & 15 & 2 & 18 & 5 & 10 & 10 & 5 & 15 & \\
\hline \multicolumn{17}{|l|}{ CHAR. Ass. } \\
\hline Juncus squarrosus & 2.4 & 2.2 & 3.5 & 3.5 & 3.5 & 2.5 & 3.5 & 3.5 & 2.3 & 2.5 & 1.3 & 3.5 & 2.5 & 3.5 & 2.5 & 100 \\
\hline Nardus stricta & 1.2 & 2.3 & 3.4 & 3.5 & 1.3 & 3.5 & 3.5 & 3.5 & 3.5 & 3.5 & 3.5 & 2.5 & 2.5 & 3.5 & 3.5 & 100 \\
\hline \multicolumn{17}{|l|}{ Nardetalia } \\
\hline Antennaria dioica & +.1 & . & . & . & . & . & . & $\cdot$ & . & $\cdot$ & . & . & . & . & . & 7 \\
\hline Arnica montana & & . & . & . & . & +.1 & . & +.1 & & +.1 & . & . & . & $\cdot$ & . & 20 \\
\hline Carex ovalis & +.1 & . & . & . & . & . & . & +.1 & +.1 & . & & . & . & 1.3 & +.1 & 33 \\
\hline Carex pallescens & +.1 & +.1 & . & . & . & . & +.1 & 1.1 & +.1 & +.1 & 1.3 & + & . & + & +.1 & 67 \\
\hline Chamaespartium sagittale & & $\cdot$ & . & . & . & . & $\cdot$ & . & $\cdot$ & . & 1.5 & . & . & . & . & 7 \\
\hline \multicolumn{17}{|l|}{ Nardo-Callunetea } \\
\hline Calluna vulgaris & 3.5 & 2.2 & 3.5 & 3.5 & 1.1 & 3.5 & 2.5 & 3.5 & 1.3 & 3.5 & 1.3 & 2.5 & 1.3 & 2.3 & 2.4 & 100 \\
\hline Danthonia decumbens & . & +.1 & . & . & +.1 & . & . & +.1 & +.1 & +.1 & 1.3 & 1.2 & 1.3 & 1.2 & 1.3 & 67 \\
\hline Deschampsia flexuosa & . & . & . & . & . & 1.1 & . & . & . & . & . & . & . & + & . & 13 \\
\hline Hieracium pilosella & +.1 & . & . & . & +.1 & . & . & +.1 & +.1 & +.1 & + & 1.3 & . & . & +.1 & 53 \\
\hline Hypericum maculatum & . & . & . & . & . & . & . & . & . & . & + & . & . & . & +.1 & 13 \\
\hline Hypochoeris radicata & . & - & . & . & . & . & . & . & +.1 & . & . & . & . & . & . & 7 \\
\hline Luzula campestris & +.1 & & . & . & . & . & +.1 & . & +.1 & . & + & . & . & . & +.1 & 27 \\
\hline Potentilla erecta & 1.1 & 1.2 & 2.3 & 1.5 & 1.2 & 1.5 & 2.5 & 1.4 & 1.3 & 1.3 & 1.3 & 1.5 & 1.5 & 1.5 & 2.5 & 100 \\
\hline Vaccinium myrtillus & . & & . & . & 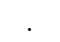 & . & . & +.1 & . & +.1 & . & . & . & . & . & 13 \\
\hline Vaccinium vitis-idaea & +.1 & . & . & 1.1 & . & +.1 & +.1 & +.1 & . & +.1 & . & . & . & . & . & 40 \\
\hline Veronica officinalis & . & . & . & +.1 & +.1 & . & . & . & +.1 & . & + & . & . & . & +.1 & 33 \\
\hline Viola canina & . & & . & . & +.1 & . & . & . & . & . & . & . & . & + & +.1 & 20 \\
\hline \multicolumn{17}{|l|}{ Caricetalia fuscae s.l. } \\
\hline Agrostis canina & . & +.1 & 1.2 & 1.1 & 2.5 & . & $\cdot$ & . & . & . & + & . & . & . & . & 33 \\
\hline Carex canescens & +.1 & +.1 & +.1 & . & . & +.1 & +.1 & +.1 & . & . & . & . & . & . & . & 40 \\
\hline Carex echinata & . & . & . & . & . & +.1 & +.1 & +.1 & . & . & . & + & + & + & . & 40 \\
\hline Carex flava & . & . & . & . & . & . & +.1 & . & . & . & . & . & . & . & . & 7 \\
\hline Carex nigra & & $\cdot$ & . & . & . & +.1 & . & . & . & . & . & + & . & . & . & 13 \\
\hline Carex oederi & +.1 & +.1 & . & . & +.1 & . & . & +.1 & +.1 & . & + & + & + & + & . & 60 \\
\hline Carex panicea & +.1 & +.1 & . & . & . & . & . & . & . & +.1 & . & . & . & . & . & 20 \\
\hline \multicolumn{17}{|l|}{ Molinietalia s.l. } \\
\hline Deschampsia caespitosa & . & . & . & . & 1.2 & . & . & . & 2.3 & 1.1 & . & 1.1 & + & + & . & 40 \\
\hline Galium palustre & . & . & . & . & . & 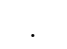 & . & . & . & . & . & + & . & . & . & 7 \\
\hline Juncus articulatus & . & . & . & . & +.1 & . & . & . & . & . & . & + & & . & . & 13 \\
\hline Juncus conglomeratus & . & - & +.1 & +.1 & 1.2 & +.1 & . & +.1 & +.1 & . & 1.2 & +.2 & 1.3 & +.3 & 1.3 & 73 \\
\hline Juncus dudleyi & . & . & . & . & . & . & . & . & . & . & 2.4 & . & 1.1 & . & & 13 \\
\hline Juncus effusus & +.1 & +.1 & +.1 & . & +.1 & . & . & +.1 & +.1 & +.1 & . & 1.2 & . & . & 1.5 & 60 \\
\hline Juncus thomasii & . & & . & . & . & . & . & . & . & . & . & . & + & . & +.1 & 13 \\
\hline Succisa pratensis & . & & . & . & . & +.1 & +.1 & . & . & . & . & . & . & + & . & 20 \\
\hline Valeriana simplicifolia & . & & . & . & . & . & . & . & . & . & + & 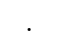 & . & . & . & 7 \\
\hline \multicolumn{17}{|l|}{ Molinio-Arrhenatheretea } \\
\hline Agrostis capillaris & . & & . & & & 1.1 & & . & 1.2 & 1.2 & . & + & 2.5 & 1.5 & 1.3 & 47 \\
\hline
\end{tabular}


Table 1. Continued.

\begin{tabular}{|c|c|c|c|c|c|c|c|c|c|c|c|c|c|c|c|c|}
\hline Relevé no. & 1 & 2 & 3 & 4 & 5 & 6 & 7 & 8 & 9 & 10 & 11 & 12 & 13 & 14 & 15 & $\mathrm{~F} \%$ \\
\hline Anthoxanthum odoratum & . & +.1 & +.1 & . & 2.5 & 1.1 & 1.3 & 1.1 & 1.2 & . & 2.5 & +.2 & + & 1.3 & 1.3 & 80 \\
\hline Cerastium holosteoides & . & . & . & . & . & . & . & . & . & +.1 & + & . & + & . & +.1 & 27 \\
\hline Cynosurus cristatus & . & . & . & . & . & . & . & . & . & . & + & . & + & . & . & 13 \\
\hline Festuca rubra & +.1 & +.1 & 1.3 & 2.3 & 2.5 & 2.5 & 2.5 & 2.5 & 3.5 & 2.4 & 1.4 & . & + & + & +.3 & 93 \\
\hline Holcus lanatus & . & +.1 & . & . & +.1 & . & . & . & . & . & + & . & + & + & 1.2 & 40 \\
\hline Leontodon autumnalis & +.1 & +.1 & . & . & +.1 & . & . & +.1 & +.1 & . & + & . & . & . & +.1 & 47 \\
\hline Leucanthemum vulgare & . & . & . & . & . & . & . & . & . & . & + & . & . & . & . & 7 \\
\hline Lotus corniculatus & . & . & . & . & . & . & . & . & . & . & + & + & . & . & . & 13 \\
\hline Plantago lanceolata & . & . & . & . & +.1 & . & . & . & . & . & . & . & . & . & +.1 & 13 \\
\hline Poa pratensis & +.1 & +.1 & . & . & . & . & . & . & +.1 & . & . & . & . & . & . & 20 \\
\hline Prunella vulgaris & . & . & . & . & 1.1 & . & . & . & . & . & + & . & + & . & . & 20 \\
\hline Ranunculus acris & . & . & . & . & . & . & . & . & . & . & + & + & + & + & . & 27 \\
\hline Stellaria graminea & . & . & . & . & . & . & . & . & . & +.1 & . & . & . & . & +.1 & 13 \\
\hline Trifolium patens & . & . & . & . & . & . & . & . & . & . & + & + & . & . & . & 13 \\
\hline Trifolium pratense & . & . & . & . & . & . & . & . & +.1 & . & . & . & . & . & . & 7 \\
\hline Trifolium repens & . & . & . & . & . & . & . & . & $\cdot$ & . & + & . & . & . & 1.3 & 13 \\
\hline \multicolumn{17}{|l|}{ OTHERS } \\
\hline Betula pendula juv. & +.1 & . & . & 1.1 & . & . & +.1 & +.1 & . & +.1 & . & + & . & + & . & 47 \\
\hline Genista tinctoria & . & . & . & . & . & . & . & . & 1.1 & . & . & . & . & . & . & 7 \\
\hline Homogyne alpina & . & $\cdot$ & . & . & $\cdot$ & . & . & . & $\cdot$ & . & . & . & . & . & + & 7 \\
\hline Juncus bufonius & . & +.1 & . & . & +.1 & . & . & . & +.1 & . & . & + & . & + & . & 33 \\
\hline Peplis portula & . & . & . & . & . & . & . & . & . & . & . & . & + & . & . & 7 \\
\hline Picea abies juv. & +.1 & . & . & . & . & +.1 & . & . & . & . & . & . & . & + & . & 20 \\
\hline Pinus sylvestris juv. & +.1 & . & . & $\cdot$ & . & . & . & . & . & . & . & . & . & . & . & 7 \\
\hline Populus tremula juv. & +.1 & . & . & +.1 & . & . & . & +.1 & . & +.1 & . & . & . & . & . & 27 \\
\hline Rumex acetosella & $\cdot$ & . & . & $\cdot$ & $\cdot$ & . & $\cdot$ & $\cdot$ & +.1 & $\cdot$ & . & . & . & . & . & 7 \\
\hline Salix aurita juv. & . & . & . & +.1 & +.1 & . & +.1 & . & . & . & . & . & . & . & . & 20 \\
\hline Salix cinerea juv. & +.1 & . & . & 1.2 & +.1 & . & +.1 & +.1 & . & +.1 & + & . & . & + & +.1 & 60 \\
\hline Sorbus aucuparia juv. & +.1 & . & . & $\cdot$ & $\cdot$ & . & $\cdot$ & $\cdot$ & . & $\cdot$ & . & . & . & . & $\cdot$ & 7 \\
\hline \multicolumn{17}{|l|}{ BRYOPHYTES } \\
\hline Ceratodon purpureus & +.1 & . & . & . & +.1 & . & . & . & +.1 & . & . & . & 1.1 & . & . & 27 \\
\hline Leucobryum juniperoideum & $\cdot$ & $\cdot$ & . & . & . & . & . & . & $\cdot$ & +.1 & . & . & . & . & . & 7 \\
\hline Pleurochaete squarrosa & . & +.1 & . & . & . & . & . & . & . & . & . & . & . & . & . & 7 \\
\hline Pleurozium schreberi & +.1 & . & . & . & . & . & . & +.1 & . & . & . & . & . & 1.2 & . & 20 \\
\hline Polytrichum formosum & +.1 & +.1 & 1.3 & +.1 & +.1 & 1.1 & . & 1.1 & . & . & 1.5 & 1.2 & 1.1 & . & 1.1 & 73 \\
\hline Polytrichum juniperinum & . & . & . & . & . & . & . & $\cdot$ & 1.1 & +.1 & . & . & . & . & 1.1 & 20 \\
\hline Sphagnum capillifolium & . & . & . & . & . & 2.3 & . & 1.1 & . & 1.3 & . & . & . & . & . & 20 \\
\hline Sphagnum compactum & 1.1 & 1.1 & 2.5 & 1.2 & . & 1.2 & 2.5 & 1.2 & . & 1.2 & . & 1.2 & . & . & 1.1 & 67 \\
\hline Sphagnum contortum & . & $\cdot$ & . & . & . & . & . & . & . & +.1 & . & . & . & . & . & 7 \\
\hline Sphagnum fallax & . & . & . & 1.1 & . & . & 1.2 & . & . & . & . & . & . & . & . & 13 \\
\hline Sphagnum palustre & . & . & +.1 & 3.5 & . & 2.5 & 2.5 & . & . & . & . & . & . & . & . & 27 \\
\hline Sphagnum rubellum & 1.1 & +.1 & 2.2 & $\cdot$ & . & 2.3 & 1.1 & . & . & 1.3 & . & . & . & . & . & 40 \\
\hline LiCHENS & + & + & + & . & . & . & . & + & + & + & . & . & . & . & . & 40 \\
\hline
\end{tabular}

\section{LOCATION OF RELEVÉS}

1-7 - Dealu Negru: at edge of Molhaşul de la Rîşca - Lágyas flat bog, 04 July 2014; 8 - Dealu Negru: on right side of DJ103K road, near Râşca Transilvană Monastery, 04 July 2014; 9 - Dealu Negru: east of Râşca Transilvană Monastery, 04 July 2014; 10 - Dealu Negru: northeast of Râşca Transilvană Monastery, 04 July 2014; 11 - Beliş: Dîmbul Negru - la Pod oligotrophic bog, 22 July 2016; 12-13 - Dealu Negru, Molhaşul de la Rîşca - Lágyas flat bog, 22 July 2016; 14-15 - Dealu Negru, Molhaşul de la Rîşca - Lágyas flat bog, 10 Aug. 2016 
strongly acidophilic flora element which grows on moist soils poor in nitrogen.

In Romania the plant communities with $J$. squarrosus (Table 1) have a different floristic composition, lacking the sub-Atlantic and Atlantic species characteristic of the communities described in Central Europe (Passarge 1964; Oberdorfer 1978) and Western Europe (McVean \& Ratcliffe 1962; Rodwell, 1991, 1992; Sýkora et al. 1993). However, one typical Atlantic bryophyte should be mentioned as present: Sphagnum compactum. This is a highly constant species (67\% frequency; Table 1) in the studied phytocoenoses. Its presence could be considered strong evidence for the indigenous origin of $J$. squarrosus in these communities. The dominant and diagnostic species for these plant communities are Juncus squarrosus, Nardus stricta and Calluna vulgaris (Table 1), covering $50-80 \%$ of the ground in the sampling plots. Besides these, there is a group of acidophilic and oligotrophic species characteristic for and indicators of nutrient-poor soils, such as Danthonia decumbens, Potentilla erecta, Carex pallescens, Carex ovalis, Arnica montana, Luzula campestris, Hypericum maculatum, Antennaria dioica and Chamaespartium sagittale (Table 1). These species, together with the dominant ones, emphasize the syntaxonomical position of phytocoenoses with J. squarrosus from Romania in the Central European syntaxonomical system (Oberdorfer 1978; Matuszkiewicz 2008): the Nardo-Callunetea Prsq. 1949 class and Nardetalia Oberd. 1949 order. We consider it more appropriate to include these phytocoenoses in Nardo-Juncion squarrosi (Oberd. 1957) Passarge 1964 alliance, together with the phytocoenoses from Central Europe (Oberdorfer 1957, 1978; Passarge 1964), although they lack some sub-Atlantic species (e.g., Polygala serpyllifolia, Pedicularis sylvatica), rather than to place them into the Violion caninae Schwickerath 1944 alliance (Matuszkiewicz 2008) which integrates hilly and mountainous grasslands with Nardus stricta (Coldea 2012).

The plant association to which the communities with heath rush from Romania were assigned is Nardo-Juncetum squarrosi (Nordh. 1920) Bük. 1942 (Table 1), which together with the
Nardo-Juncion squarrosi (Oberd. 1957) Passarge 1964 alliance are new syntaxa for the Romanian vegetation.

The floristic composition of the phytocoenoses with $J$. squarrosus in Europe varies, mainly depending on the region where they grow and on the climate. In this respect the Western and Central European communities have in their structure some Atlantic species (Erica tetralix, Narthecium ossifragum, Carex binervis, Trichophorum caespitosum subsp. germanicum) that are not present in the floristic composition of the Eastern European communities. Therefore the phytocoenoses with $J$. squarrosus are published in scientific studies not only as a distinct plant community, Juncus squarrosus-Festuca ovina (Rodwell 1992), but also as plant associations named Juncetum squarrosi sub-alpinum (McVean \& Ratcliffe 1962), Lycopodio-Rhynchosporetum (Sýkora et al. 1993) and Juncetum squarrosi (Oberdorfer 1978; Krahulec et al. 2007). The names of these syntaxa lack relevant persuasive phytogeographical arguments, and for this reason Passarge (1964) considered the syntaxon Juncetum squarrosi Nordh. 1923 to be a group of three associations: $(i)$ the boreo-Atlantic association Genisto anglicae-Juncetum squarrosi Passarge 1964; (ii) the mountain-Atlantic association Polygalacto serpyllifolii-Juncetum squarrosi (Oberd. 1934) Büker 1942, where he separated several geographical races; and (iii) the northwestern association Rhytidiadelpho-Juncetum squarrosi Br.-B1. \& Tx. 1952, specific to Ireland.

Because the phytocoenoses with $J$. squarrosus described in Central Europe (Zarzycki 1958; Passarge 1964: tab. 88, col. d-g; Krahulec et al. 2007) and those from Romania presented in this study have a similar floristic composition, they should be included in a unique association. The association name Nardo-Juncetum squarrosi (Nordh. 1920) Bük. 1942, used by Matuszkiewicz (2008) for the phytocoenoses from Poland, is considered appropriate from the floristic point of view for all the communities with $J$. squarrosus in Central and Eastern Europe.

ACKNOWLEDGEMENTS. We are grateful to the anonymous reviewers for helpful remarks and suggestions on the 
manuscript, and to Mihaela-Felicia Oanea for editing the English of the manuscript for submission. The field work of the first author was financially supported under the Sectoral Operational Programme (SOP) Environment: Monitoring of conservation status of the species and habitats in Romania under Article 17 of the Habitats Directive (SMIS-CSNR 17655).

\section{REFERENCES}

ANDRIENKo T. L. 1983. Central-European species of the genus Juncus: J. squarrosus, J. bulbosus (Juncaceae) in the Ukraine. Bot. Zhurn. 68(5): 644-648 (in Russian).

AnONYmous 2006-2017. Juncus squarrosus L. In: Euro+Med PlantBase. [15 Oct. 2017]. http://ww2.bgbm.org/EuroPlusMed/.

Baumgarten J. CH. G. 1816. Enumeratio Stirpium Magno Transsilvaniae Principatui praeprimis indigenarum in usum nostratum botanophilorum conscripta inque ordinem sexuali-naturalem concinnata. 3. Libraria Camesinae, Vindobonae.

Borza A. 1934. Études phytosociologiques dans les monts du Rétézat. Bul. Grad. Bot. Univ. Cluj 14(1-2): 1-84 (in Romanian with French summary).

Borza A. 1947. Conspectus Florae Romaniae Regionumque Affinium. Instituti Botanici Universitatis Clusiensis, Cluj.

Braun-Blanquet J. 1928. Pflanzensoziologie. Grundzüge der Vegetationskunde. Springer-Verlag, Berlin.

Braun-Blanquet J. 1964. Pflanzensoziologie. Grundzüge der Vegetationskunde. Springer Verlag, Wien - New York.

Chifu T., MÂNZU C. \& ZAMFIRESCU O. 2006. Flora şi vegetaţia Moldovei (România). I. Flora. Universitatea "Al. I. Cuza", Iaşi.

CIOCÂRLAN V. 2000. Flora ilustrată a României. Pteridophyta et Spermatophyta. Ceres, Bucureşti.

CiocÂRLAN V. 2009. Flora ilustrată a României. Pteridophyta et Spermatophyta. Ceres, Bucureşti.

Coldea G. 2012. Les associations végétales de Roumanie. Les associations antropogènes. 2. Presa Universitară Clujeană, Cluj-Napoca.

Ellenberg H. 1979. Zeigerwerte der Gefäßpflanzen Mitteleuropas. Scripta Geobotanica 9: 7-122.

Frink J. P., JAKAB G. \& SASS-Gyarmati A. 2014. The first certain record of Juncus squarrosus L. (Juncaceae) in the Romanian flora. In: Floristic patterns at different organisation and distribution levels, Conference Abstracts, pp. 20-21. Cluj-Napoca.

GRINŢESCU I. 1966. Juncus L. In: T. SăVULESCU \& E. I. NYÁRÁDY (eds), Flora Republicii Socialiste România. 11: 528-578. Academia Republicii Socialiste România, Bucureşti.
Hill M. O., Bell N., Bruggemann-Nannenga M. A., Brugués M., Cano M. J., Enroth J., Flatberg K. I., Frahm J.-P., Gallego M. T., Garilleti R., Guerra J., Hedenäs L., Holyoak D. T., Hyvönen J., Ignatov M. S., Lara F., Mazimpaka V., MuŇoz J. \& Söderström L. 2006. An annotated checklist of the mosses of Europe and Macaronesia. J. Bryol. 28: 198-267.

Jávorka S. 1925. Magyar Flóra (Flora Hungarica). Magyarország virágos és edényes virágtalan növényeinek meghatározó kézikönyve. Studium, Budapest.

Krahulec F., ChytrÝ M. \& Härtel H. 2007. Calluno-Ulicetea. In: M. ChytrÝ (ed.), Vegetace České Republiky. Travinná a keřičková vegetace. 1: 281-320. Academia, Praha.

MatuszKiewicz W. 2008. Przewodnik do oznaczania zbiorowisk roslinnych Polski. Wydawnictwo Naukowe PWN, Warszawa.

McVean D. N. \& Ratcliffe D. A. 1962. Plant communities of the Scottish Highlands. A study of Scottish mountain, moorland and forest vegetation. William Clowes and Sons Limited, London.

Meusel H., Jäger E. \& Weinert E. 1965. Vegleichende Chorologie der Zentraleuropaischen Flora. VEB Gustav Fischer Verlag, Jena.

NeILreich A. 1866. Aufzählung der in Ungarn und Slavonien bisher beobachteten Gefässpflanzen, nebst einer pflanzengeographischen Übersicht. Wilhelm Braumüller, Wien.

Oberdorfer E. 1957. Süddeutsche Pflanzengesellschaften. VEB Gustav Fischer Verlag, Jena.

OBERDORfER E. 1978. Süddeutsche Pflanzengesellschaften. Pflanzensoziologie. 2. Auflage. 10 (2). VEB Gustav Fischer Verlag, Jena.

Oprea A. 2005. Lista critică a plantelor vasculare din România. Universitatea "Al. I. Cuza", Iaşi.

Passarge H. 1964. Pflanzengesellschaften des norddeutschen Flachlandes. I. Pflanzensoziologie. 13. VEB Gustav Fischer Verlag, Jena.

PoP E. 1947. Angaben über Hochmoore und moorpflanzen aus Rumänien. III. Die moore der Gegend Calatele. Bul. Grad. Bot. Univ. Cluj 27: 65-79 (in Romanian with German summary).

PoP E. 1960. Mlaştinile de turbă din Republica Populară Romînă. Academia Republicii Populare Romîne, Bucureşti.

Pop G. 1962. Histoire morphogénétique de la vieille surface d'érosion "Fărcaș" des monts de Gilău (M. Apuseni). Studia Universitatis Babeş-Bolyai. Series Geologia-Geographia 1: 89-110 (in Romanian with French summary).

Prodan I. 1939. Flora pentru determinarea şi descrierea plantelor ce cresc în România. Ediţia a Doua. 1. Cartea Românească, Cluj.

Rodwell J. S. (ed.) 1991. British Plant Communities. Mires and heaths. 2. Cambridge University Press, Cambridge. 
Rodwell J. S. (ed.) 1992. British Plant Communities. Grasslands and montane communities. 3. Cambridge University Press, Cambridge.

Rodwell J. S., Schaminée J. H. J., Mucina L., Pignatti S., DrING J. \& Moos D. 2002. The diversity of European vegetation. An overview of phytosociological alliances and their relationships to EUNIS habitats. National Reference Centre for Agriculture, Nature and Fisheries, Wageningen.

SÂrbu I., ŞTEFAn N. \& OpreA A. 2013. Plante vasculare din România. Determinator ilustrat de teren. Victor B Victor, Bucureşti.

Simonkai L. 1886. Erdély edényes flórájának helyesbitett foglalata (Enumeratio Florae Transsilvanicae vesculosae critica). Királyi Magyar Természettudományi Társulat, Budapest

Simonkai L. 1890. Nagyváradnak és vidékének növényvilága. In: V. BunYITAY (ed.), Nagyvárad Természetrajza, pp. 45-134. Franklin-Társulat, Budapest.

Snogerup S. 1980. Juncus L. In: T. G. Tutin, V. H. Heywood, N. A. Burges, D. M. Moore, D. H. Valentine, S. M. WalTERS, D. A. WeBb (eds), Flora Europaea. 5: 102-111. Cambridge University Press, Cambridge.

Soó R. 1940. A Székelyföld flórájának elömunkálatai. Prodromus Florae Terrae Siculorum (Transsilvaniae Orientalis). Instituti Syst.-Geobotanici Museique Botanici Universitatis Kolozsvar, Kolozsvár.
STEFFEK A. 1864. Übersicht der bei Grosswardein bis jetzt beobachteten Phanerogamen. Oesterr. Bot. Z. 14(6): 169-187.

SÝkora K. V., NiJs L. J. \& Pelsma T. A. H. M. 1993. Plantengemeenschappen van Nederlandse wegbermen. III. 59. Stichting Uitgeverij Koninklijke Nederlandse Natuurhistorische Vereniging, Utrecht.

Tutin T. G., Heywood V. H., Burges N. A., Moore D. M., Valentine D. H., Walters S. M. \& Webb D. A. (eds) 1964-1980. Flora Europaea. 1-5. Cambridge University Press, Cambridge.

Welch D. 1964. Studies in the autecology of Juncus squarrosus L. Durham Theses, Durham University, Durham.

Welch D. 1966a. Biological Flora of the British Isles: Juncus squarrosus. J. Ecol. 54(2): 535-548.

Welch D. 1966b. The reproductive capacity of Juncus squarrosus. New Phytol. 65: 77-86.

ZARZYCKI K. 1958. Die wichtigsten Grünlandgesellschaften des oberen Weichseltales und die Grundwasser Ganglinien. Acta Soc. Bot. Poloniae 27(3): 383-428 (in Polish with German summary). 